\title{
Genome-wide identification and characterization of transfer RNA-derived small RNAs in Plasmodium falciparum
}

\author{
Zhensheng Wang ${ }^{1}$, Chunyan Wei ${ }^{1}$ Xiao Hao ${ }^{1}$, Weiwei Deng ${ }^{1}$, Lianhui Zhang ${ }^{1}$, Zenglei Wang ${ }^{2 *}$ and Heng Wang ${ }^{1 *}$
}

\begin{abstract}
Background: Transfer RNA (tRNA)-derived fragments (tRFs) have been widely identified in nature, functioning in diverse biological and pathological situations. Yet, the presence of these small RNAs in Plasmodium spp. remains unknown. Systematic identification and characterization of tRFs is therefore highly needed to understand further their roles in Plasmodium parasites, particularly in the virulent Plasmodium falciparum parasite.

Results: Genome-wide small RNAs with sizes ranging from 18-30 nucleotides from $P$. falciparum were deepsequenced via Illumina HiSeq 2000 technology. In-depth analysis revealed the presence of a vast number of small RNAs originating from tRNA-coding genes, responsible for $22.4 \%$ of the total reads as the second predominant group. Three $P$. falciparum-derived tRF types (ptRFs) were identified as 5'ptRFs, mid-ptRFs and 3'ptRFs. The majority (90\%) of ptRFs were derived from tRNAs that coded eight amino acids: Pro, Phe, Asn, Gly, Cys, Gln, His and Ala. Stem-loop reverse transcription polymerase chain reaction further confirmed the presence of tRFs in the blood stages of $P$. falciparum. Four new motifs with an enriched $\mathrm{G} / \mathrm{C}$ feature were determined at cleavage sites that might guide the generation of ptRFs.
\end{abstract}

Conclusions: To our knowledge, this is the first report of a genome-wide investigation of ptRFs from Plasmodium species. The identification of ptRFs reveals a complex small RNA system manipulated by the malaria parasite, and might promote research on the function of tRFs in the pathogenesis of Plasmodium infections.

Keywords: Plasmodium falciparum, Transfer RNA, Short RNAs, Small RNAs, tRFs

\section{Background}

A growing amount of data suggests that small RNAs play critical regulatory roles in many biological processes in both prokaryotes [1-3] and eukaryotes $[4,5]$. Other than the three major types of small RNAs including microRNAs (miRNAs), endogenous small interference RNAs (endo-siRNAs) and PiWi-interacting RNAs (piRNAs) [6, 7], the advance in deep sequencing technology has unveiled new classes of small RNAs with novel features and functions. One such class is those derived from transfer RNAs (tRNAs), commonly referred to as

\footnotetext{
* Correspondence: zengleiwang@pumc.edu.cn; wangh@ibms.cams.cn ${ }^{2} \mathrm{NHC}$ Key Laboratory of Systems Biology of Pathogens, Institute of Pathogen Biology, Chinese Academy of Medical Sciences and Peking Union Medical College, Beijing, People's Republic of China

'Department of Microbiology and Parasitology, Institute of Basic Medical Sciences Chinese Academy of Medical Sciences, School of Basic Medicine Peking Union Medical College, 5\# Dong Dan San Tiao, Beijing 100005, People's Republic of China
}

tRFs (tRNA-derived RNA fragments), which were once mis-annotated as miRNAs $[8,9]$.

tRFs are typically short in length, ranging from $12-30$ nucleotides (nt) [10]. Despite the fact that the nomenclature used to refer to the subclass of tRFs varies in the literature, they are generally classified in terms of their origin: 5 'tRFs are generated from $5^{\prime}$ end cleavage in the D-arm of mature tRNAs, 3'tRFs or 3'CCA tRFs are produced from 3 ' end processing of mature tRNAs in the TYC-arm and contain a CCA post-transcriptional modification, 3 'UtRFs or tRF- 1 are derived from the 3 ' end of a pre-tRNA, and endogenous tRFs (itRFs or mid-tRFs) originate from a combination of cleavage from the anticodon loop domain and either D-arm or T $\Psi \mathrm{C}$-arm of mature tRNAs. Single cleavage occurring in the anticodon loop could lead to generation of a conventional class of tRNA-derived RNAs, namely tRNA-halves ( 30-50 nt). Apart from the yield of tRNA-halves from mature tRNA 
under stress conditions, the biogenesis of tRFs as well as their functions remain to be fully understood.

With increased research interest in tRFs, mounting evidence has linked the production of tRFs to cancer [11], neurodegenerative disorders, viral infection and other pathological conditions. They have been involved in cellular proliferation, RNA interference pathway and regulate gene translation at the post-transcriptional level, similar to miRNAs (reviewed in [10, 12]). Moreover, the interactions in many cases between tRFs with the functional proteins in miRNA pathway such as argonaute (AGO) protein families [13, 14], PIWI [15] and DICER proteins [16] further imply the possibility of tRFs behaving like miRNAs and siRNAs.

Malaria remains the most grievous protozoan load due to its severe prevalence and lethality in the world, particularly in Southeast Asia and Africa. More than 400,000 deaths were recorded in 2017 by the WHO Malaria Report [17]. Of the five human malaria parasite species, Plasmodium falciparum is the most virulent, causing the majority of malaria-related mortality. In P. falciparum, as well as in other Plasmodium parasites, active RNA interference apparatus [18] and canonical miRNA molecules were found to be absent $[19,20]$, as confirmed by computational and experimental evidence. The lack of DICER and AGO homologs in Plasmodium parasites suggests that these organisms might use an alternative pathway to accomplish the intense post-transcriptional regulation needed during the rapid morphological change in the intraerythrocytic cycle. It is possible that small RNAs such as tRFs might be involved. Until now, the existence and characterization of tRFs in Plasmodium parasites has not been evaluated. tRFs have been identified in several protozoan organisms including Tetrahymena [21], Giardia lamblia [22], Trypanosoma cruzi [23] and the exosomes from Leishmania donovani [24]. This provides credence that these deeply conserved molecules are expressed in protozoan species, even though their classifications and functions remain unclear. With this inspiration, we sought to investigate tRFs in the malaria parasite, in which the machinery associated with small RNA biogenesis is thought to be either entirely lost or extensively simplified [18-20].

Here, we focused on the deadly P. falciparum and took the advantage of deep sequencing technology to comprehensively analyze genome-wide high-throughput sequencing data from P. falciparum-derived small RNAs. To our knowledge, we report for the first time the expression of novel, endogenous Plasmodium tRFs (ptRFs) and their global distribution and characterization in blood stages of $P$. falciparum.

\section{Methods}

\section{Maintenance of $P$. falciparum}

The standard $P$. falciparum laboratory strain 3D7 (Pf3D7) was obtained from ATCC (Manassas, VA, USA) and maintained within fresh human red blood cells (RBCs) at 5\% hematocrit in RPMI-1640 medium supplemented with $0.5 \%(\mathrm{w} / \mathrm{v})$ albumax II (Invitrogen, Carlsbad, CA, USA), as previously described [25]. Parasitemia was monitored by Giemsa staining of blood smears.

\section{Small RNA purification and high-throughput sequencing}

The mixed-stage infected RBCs were lysed with $0.05 \%$ saponin and washed three times with RPMI-1640 to collect parasites. Total RNA from the parasite pellet was extracted using Trizol reagent (Invitrogen) following the manufacturer's instructions and was quantified by absorbance at $260 \mathrm{~nm}$. RNA was then separated by urea-denatured $15 \%$ polyacrylamide gel electrophoresis, and bands of small RNAs of 18-30 nt in length were extracted and purified. Small RNA library construction and sequencing was performed following commercial protocols. Briefly, the small RNA molecules were ligated to $5^{\prime}$ and $3^{\prime}$ adaptors sequentially and then converted to cDNA with reverse transcription followed by polymerase chain reaction (PCR) amplifications. Approximately 20 $\mu \mathrm{g}$ of reverse transcription (RT)-PCR products per sample were sequenced using an Illumina Hiseq 2000 platform (Illumina, San Diego, CA, USA) by Beijing Genomics Institute (BGI, Shenzhen, China).

\section{Small RNA data analysis}

A total of 14,828,877 reads were initially obtained from the sequencing of small RNA libraries. Raw sequences were pre-cleaned to remove low-quality reads and $10,230,166$ high-quality reads were then trimmed to discard the $3^{\prime}$ and $5^{\prime}$ adaptors, sequences containing the polyA tail, and those smaller than $18 \mathrm{nt}$ or larger than $30 \mathrm{nt}$. This dataset was mapped to the genome of the $P$. falciparum 3D7 strain (PlasmoDB, release 36, http:// plasmodb.org/plasmo/) using BLASTN to allow the removal of any contamination from human RNA segments to yield a clean data pool. These data were analyzed in both total and unique aspects. Total reads, also referred to as redundant reads in the literature, indicated multiple reads aligned around the same location, representing high abundance of the RNA. To demonstrate the diversity of the small RNA, the total reads were collapsed to remove the redundant ones, resulting in single consensus aligned unique reads. The clean-read counts were normalized as a relative number per one million reads (RPM) in both total and unique categories for further analysis. Information of the 45 P. falciparum tRNA genes was downloaded from the PlasmoDB database. By performing BLAST alignments, data from the mapped Plasmodium tRNA-derived segments were extracted and further analyzed. The expression intensity of the ptRFs against the whole genome was sequentially calculated with the "Build" function of Bowtie2 (v.2.1.0.0) and the pipeline 
of TopHat (v.2.0.9) and Cufflinks (v.2.2.1). The data extraction of each type ptRFs was performed by compiled Perl (v.5.22) codes. The statistical analysis for the Pearson's correlation coefficients was performed using the "cor" function in $\mathrm{R}$ package stats. The plots were drawn mainly with the ggplot2 package in R language (v.3.5.0).

\section{Motif analysis}

Gapped Local Alignment of Motifs (GLAM2 v1056) [26] and Motif Comparison Tool (Tomtom v.4.12.0) [27] were used to predict and compare the motifs around the cleavage sites of the tRFs. For the GLAM2 algorithm, the adopted parameters for motif predication were set as default values, except that only the given strand was aligned. The outputs were directly submitted into the Tomtom algorithm for comparing the predicted motifs against the known RNA motif database. All known motifs were selected to be calculated using an e-value of $<10$ followed by the adoption of the function for Pearson's correlation coefficient.

\section{Stem-loop RT-PCR}

Total RNA templates were reverse-transcribed into cDNAs using the EasyScript One-Step gDNA Removal and cDNA Synthesis SuperMix Kit (TransGen Biotech, Beijing, China) according to the manufacturer's instructions. Specific stem-loop reverse transcription primers (Additional file 1: Table S1) were designed based on the sequence of each ptRFs as described previously $[28,29]$. The PCR cycling conditions were as follows: initial denaturation at $94{ }^{\circ} \mathrm{C}$ for $5 \mathrm{~min}$, followed by 30 cycles of denaturation at $94{ }^{\circ} \mathrm{C}$ for $5 \mathrm{~s}$, annealing at $50{ }^{\circ} \mathrm{C}$ for $15 \mathrm{~s}$ and extension at $72{ }^{\circ} \mathrm{C}$ for $15 \mathrm{~s}$, with a final extension at $72{ }^{\circ} \mathrm{C}$ for $5 \mathrm{~min}$. The PCR products were separated on $12 \%$ polyacrylamide gel. The expected sizes of the PCR products were calculated by the length of each ptRF plus 40 base pairs of nucleotides technically introduced in the design of specific stem-loop primers. Negative controls, absent with either DNase I-treated RNA or reverse transcriptase in RT reactions, were performed to verify the accuracy and specificity of the stem-loop RT-PCR. The sequences of RT-PCR primers are listed in Additional file 1: Table S2.

\section{Results}

General features of tRNA-derived small RNAs in $P$. falciparum The quality control strategies gave rise to a total of $9,767,982$ high-quality clean reads ranging from 18 to 30 nt in length. Of these, $75.7 \%$, equivalent to $7,392,533$ reads, were perfectly mapped to the nuclear genome, whereas 0.09 and $1.72 \%$ matched to the apicoplast and mitochondrial sequences, respectively (Table 1). Sequences without genomic match were assumed to be host-derived small RNAs, which might be erythrocytic
Table 1 Classification of small RNAs mapping to the $P$. falciparum genome

\begin{tabular}{lll}
\hline Categories & Total & Unique \\
\hline Clean reads & $9,767,982$ & 957,443 \\
Unmapped reads & $2,198,260$ & 312,761 \\
Nuclear genome & $7,392,533$ & 635,476 \\
mRNAs & 634,793 & 422,374 \\
rRNAs & $4,464,246$ & 130,442 \\
tRNAs & $2,184,619$ & 28,091 \\
snRNAs/snoRNAs & 110,769 & 8,287 \\
Annoted ncRNAs & 95,718 & 7107 \\
Unannoted small RNAs & 80,444 & 39,175 \\
Apicoplast & 9298 & 2415 \\
Mitochondria & 167,891 & 6791 \\
\hline
\end{tabular}

miRNAs translocated into the parasite cytosol as previously reported [30].

The distribution of these small RNA reads appeared to be normal in length, with a peak at $23 \mathrm{nt}$ for both total and unique RNAs, accounting for $12 \%$ of the total small RNAs and $11 \%$ of the unique RNAs, respectively (Fig. 1a). However, the slope of this peak is much lower than that for Toxoplasma gondii, an evolutionarily close apicomplexan parasite. Within the same range of 18-30 nt, small RNAs in T. gondii reached peaks at 21 and 26 nt in two different strains, in correspondence to $18.92 \%$ and $18.13 \%$ of unique small RNAs, respectively [31]. This implied that the features of $P$. falciparum small RNAs seemed to be different from the canonical 21-23 nt miRNAs. These P. falciparum small RNAs were derived from mRNAs, ribosomal RNAs (rRNAs), tRNAs, small nuclear RNA (snRNA)/small nucleolar RNAs (snoRNA), previously reported non-coding RNAs (ncRNAs), as well as some unannotated RNAs (Table 1, Fig. 1b). As expected, we found that the tRNA-derived small RNAs were abundantly presented in the library as the second predominant group, which represented $22.4 \%$ of the total reads (Fig. 1b). These small RNAs prevailed on all tRNA-originated chromosomes, while they were not found on chromosomes $1,8,9$ and 10 due to the absence of tRNA genes on these chromosomes (Fig. 1c). Since only RNAs with a size of 18-30 nt were included in this study, shorter than those of mature tRNAs or tRNA-halves, and since quality control before sequencing limited their possibility of being RNA degradation residues during RNA processing, we predicted these tRNA-derived reads were Plasmodium tRFs.

\section{Identification of three types of tRFs from $P$. falciparum}

We evaluated the amino acids coded by parental tRNAs of these ptRFs. The parental tRNAs of these ptRFs coded a total of 20 amino acids; however, $90 \%$ of the ptRFs 

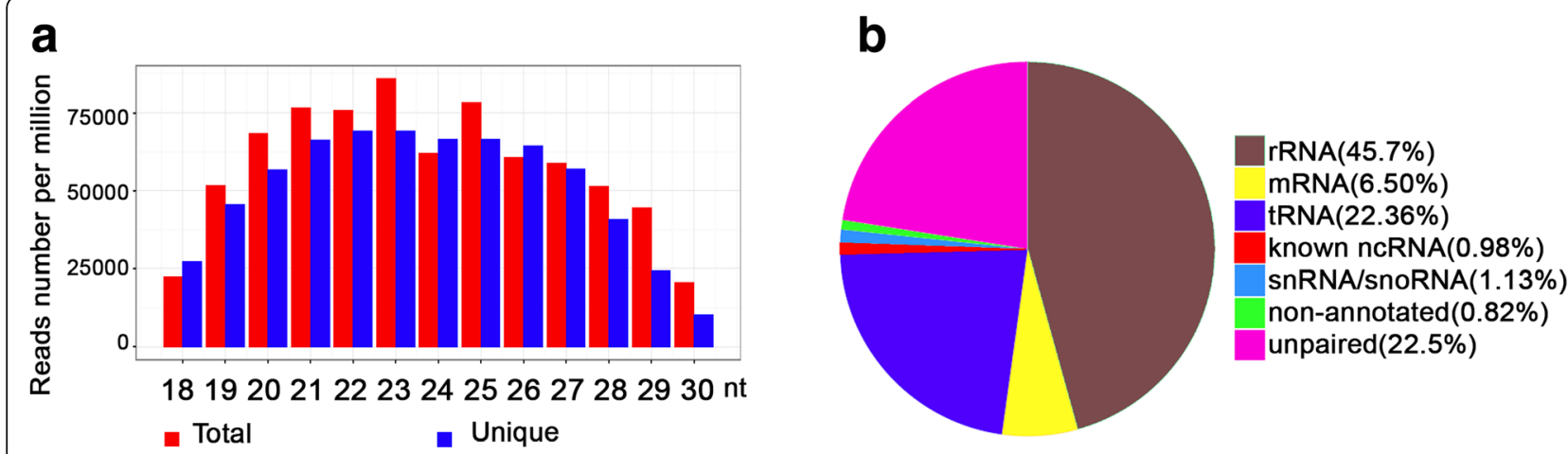

C

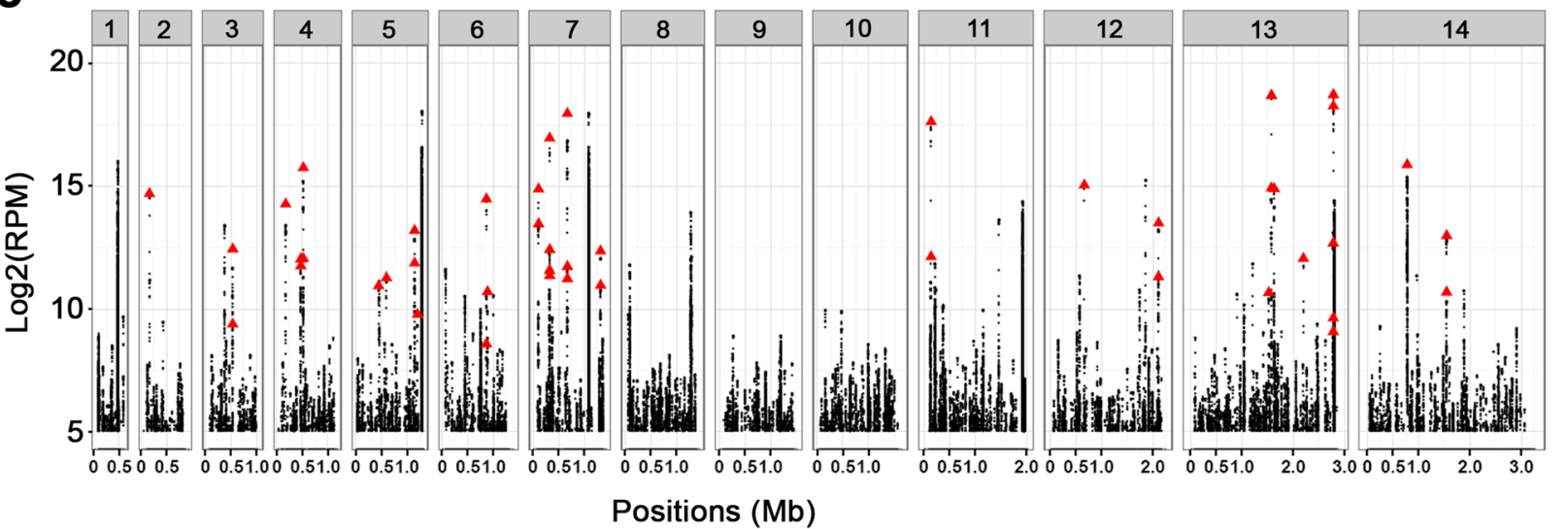

Fig. 1 General features of 18-30 nt length small RNAs from P. falciparum. a Size distribution of the small RNAs derived from blood-stage parasites. $\mathbf{b}$ Classification and percentage of small RNA populations referring to the $P$. falciparum genome. $\mathbf{c}$ Schematic representation of the genome-wide density analysis for small RNAs on $P$. falciparum chromosomes. The read counts of total small RNAs were normalized to the relative number per one million reads (RPM) and plotted against the 14 parasite chromosomes. Red triangles show the locations and abundance of the P. falciparum tRNA-derived reads

were derived from tRNAs that coded eight amino acids: Pro, Phe, Asn, Gly, Cys, Gln, His and Ala (Fig. 2a). This bias further proved that these RNA fragments were not produced by random degeneration of mature tRNAs. We analyzed the RPM values of these fragments against their sizes and identified a distinct pattern in their size distribution, which showed two prominent peaks at $\sim 23$ and $\sim 29$ nt in correspondence to two groups (18-25 nt and 26-30 nt, respectively) (Additional file 2: Figure S1a).

Given that the biogenesis of tRFs from their parental tRNA molecules was reported to be evolutionarily conserved at the $5^{\prime}$ end, $3^{\prime}$ end and tRNA precursor, we mapped the ptRF reads to all 45 Plasmodium tRNA-coding genes and, as expected, we observed that a large portion of the reads fell into two groups that mapped to the $5^{\prime}$ and $3^{\prime}$ ends of their parent tRNAs. Unexpectedly, it was noticed that part of the ptRFs were mapped to the middle regions of the parent tRNAs, which were divergent in size and ends in contrast to the known mid-tRFs (or itRFs). Hence, we named these three ptRF types as "5'ptRFs", "3'ptRFs" and "mid-ptRFs".
5 'ptRFs predominated (86.23\%) in ptRFs, while mid-ptRFs and 3'ptRFs represented only $6.20 \%$ and $5.99 \%$ of the tRNA-derived fragments, respectively (Fig. 2b). By plotting the RPM values of three types of ptRFs against their sizes, we observed two peaks at $~ 23 /$ 24 and $\sim 29$ nt for 5 ptRFs, one peak at $\sim 20$ nt for mid-ptRFs, and two peaks at $\sim 20 / 21$ nt and $\sim 25$ nt for 3'ptRFs (Fig. 2c), which displayed great difference in the expression levels.

To further understand their expression divergence, we investigated the abundance of ptRFs derived from all 45 P. falciparum tRNAs. The RPM values of ptRFs in both total and unique categories were plotted against their sizes to create 270 plots (Additional files 3, 4 and 5: Figures S2, S3, S4), which exhibited great variance of the ptRFs from different tRNAs. Nine ptRFs with high RPM values over $10^{5}$ were observed, namely 5 'ptRFs-Asp ${ }^{\mathrm{GTC}}$, $-{ }^{-C y s}{ }^{G C A},-G l y^{G C C},-\mathrm{His}^{\mathrm{GTG}},-\mathrm{Leu}^{\mathrm{CAA}},-\mathrm{Pro}^{\mathrm{AGG}}$, -Pro ${ }^{\mathrm{CGG}}$, -Pro ${ }^{\text {TGG }}$ and 3 'ptRFs-Asp ${ }^{\text {GTC; }}$; whereas four ptRFs, 5 'ptRFs-Sec ${ }^{\text {TCA }}$, mid-ptRFs-Arg ${ }^{\text {TCG }}$, - Leu $^{\text {AAG }}$ and - Leu $^{\text {TAG }}$ were found to have low RPM values less than 5 


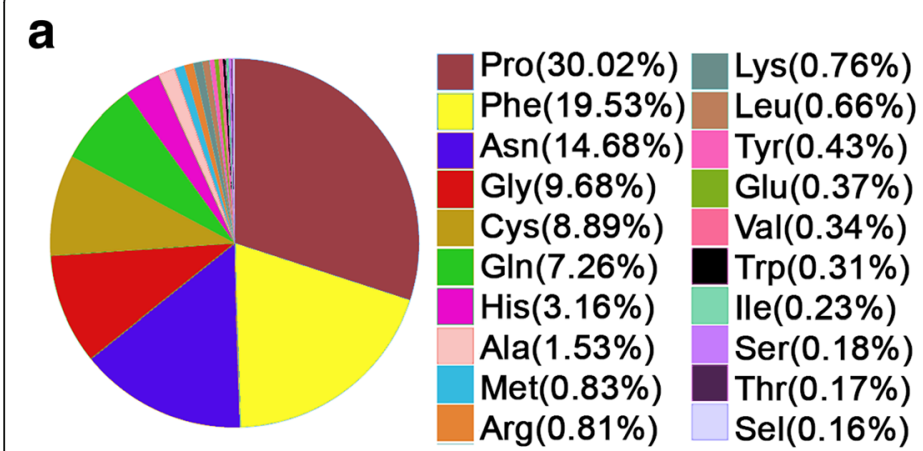

C

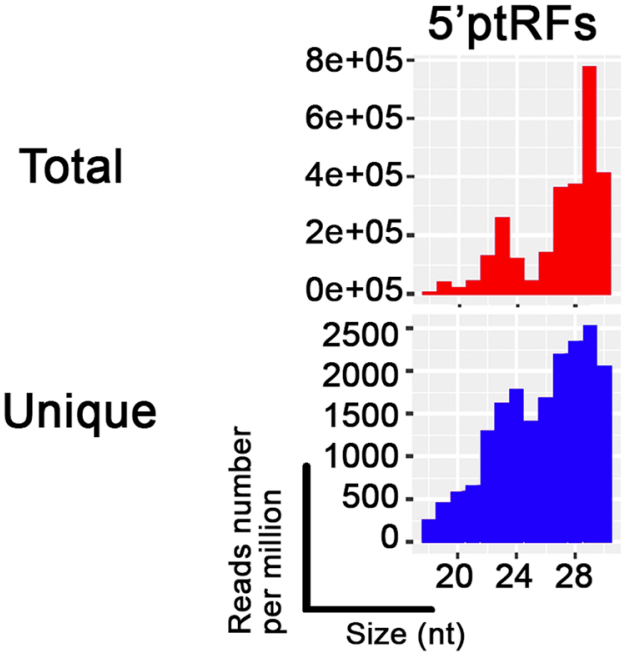

d
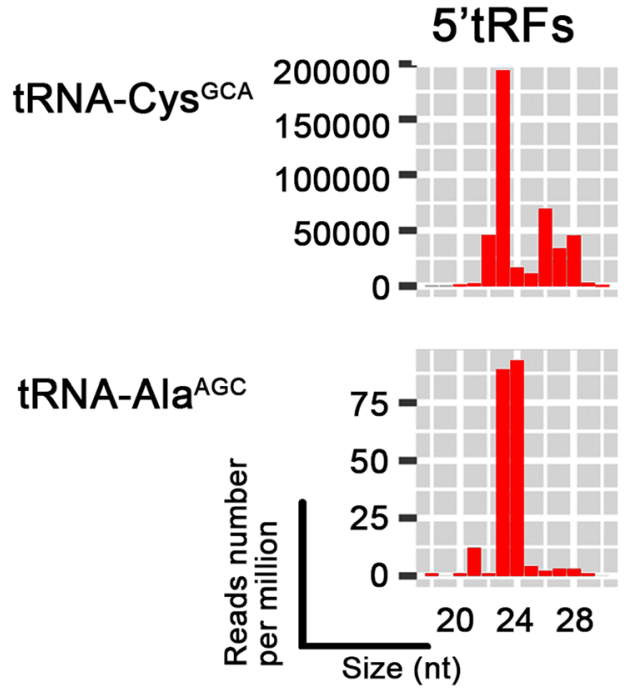

b

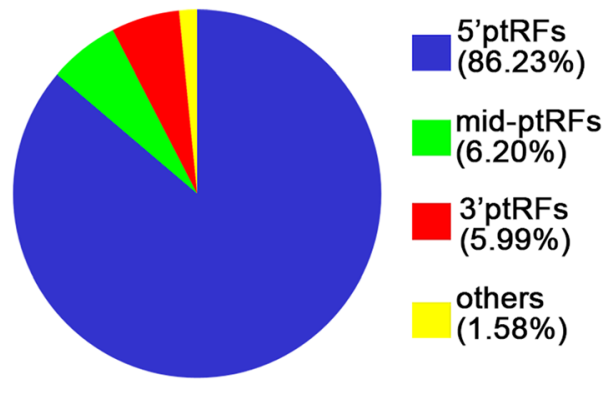

5'ptRFs mid-ptRFs

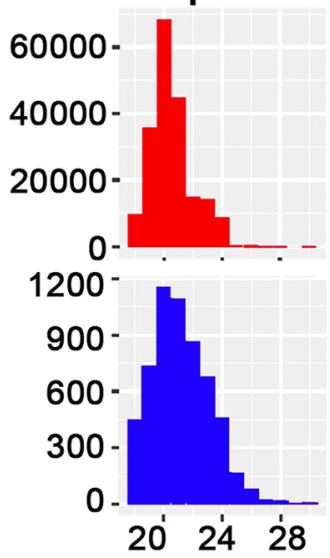

mid-tRFs
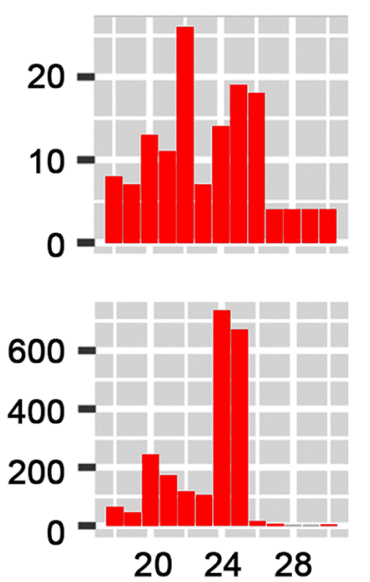

3'ptRFs

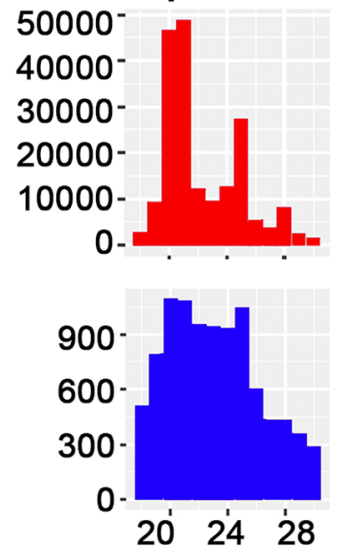

\section{3'tRFs}
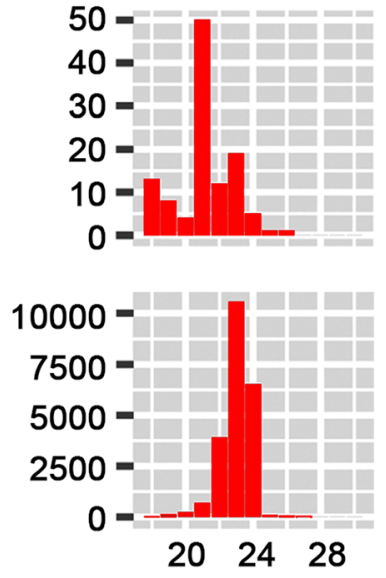

Fig. 2 Distribution and abundance of 5'ptRFs, mid-ptRFs and 3'ptRFs. a ptRFs-originated tRNAs. b Frequency of ptRFs generated by tRNAs. c Size distribution of three ptRF types analyzed for their total and unique aspects. $\mathbf{d}$ Abundance analysis of three ptRF types derived from tRNA-Cys ${ }^{G C A}$ and tRNA-Ala ${ }^{A G C}$

(Additional files 3, 4 and 5: Figures S2, S3, S4). For a given tRNA gene, the production of three types of ptRFs varied dramatically. For example, tRNA-Cys ${ }^{\mathrm{GCA}}$ produced more 5 'ptRFs than mid-ptRFs or 3'ptRFs with an abundance $3 \times$ $10^{3}$-fold higher, while tRNA-Ala ${ }^{\mathrm{AGC}}$ generated a lower level of 5'ptRFs than mid-ptRFs or 3'ptRFs (Fig. 2d). Consequently, no significant correlation was found within the abundance of these three types in the library. The Pearson's correlation coefficient was $-0.0176(P=0.6275)$ between 5'ptRFs and mid-ptRFs, 0.3192 between mid-ptRFs 
and 3'ptRFs $(P=0.3151)$, and 0.0218 between 3'ptRFs and 5 ptRFs $(P=0.8068)$ (Additional file 2: Figure S1b). We then selected the top 20 abundant ptRFs of each type to avoid any possibility of them being as RNA degradation products for further analysis (Additional file 1: Table S3). Interestingly, these three types of ptRFs were generally derived from various tRNAs, sharing only four common parental tRNAs (Additional file 2: Figure S1c). The generation of ptRFs did not seem to be associated with the expression levels of their parental tRNAs in P. falciparum.

\section{Experimental validation of ptRF candidates}

We experimentally evaluated the expression of the ptRFs in the transcriptome of $P$. falciparum to prove our findings. The top 20 abundant ptRFs of each type corresponding to 60 candidates were selected for stem-loop reverse transcription polymerase chain reaction (stem-loop RT-PCR). Thirty-five of these candidates (10 in 5 'ptRFs, 14 in mid-ptRFs, and 11 in 3'ptRFs) were specifically amplified (Fig. 3), accounting for $58.3 \%$ of tested ptRFs. This confirmed the presence of tRFs in the blood stages of $P$. falciparum.

New motifs found to process ptRFs at their cleavage sites To identify the cleavage sites, we matched each ptRF to their parental tRNA. As displayed in Fig. 4a, two cleavage sites were discovered to generate 5 'ptRFs, with one occurring at the D-arm to produce 5'ptRFs of $23 / 24$ nt, while one taking place at the anticodon stem to give rise to those with sizes around $29 \mathrm{nt}$. The mid-ptRFs originated from a combination of cleavage in the anticodon loop domain and $\mathrm{T} \psi \mathrm{C}$ arm. However, the anticodon loop domain was cleaved at the 5 ' side instead of the middle section in this species, which was novel. Consequently, the cleavage in $\mathrm{T} \psi \mathrm{C}$ arm generated the 3'ptRFs, and the variation of 3 'CAA end in tRNAs resulted in two size groups (3'ptRFs-20/21 nt and 3'ptRFs-25 nt). We further explored motifs at the cleavage sites that might guide the generation of the ptRFs. Four motifs (motifs 1-4) were predicted to process ptRFs at each cleavage site (Fig. 4b). Although these motifs varied in sequence, they all had a signature of enriched G/C at the cleavage sites (Fig. 4b). Despite the fact that the 5'ptRFs showed two sub-classes, one common motif seemed to be responsible for the cleavage. Through comparison analysis, we sought to determine whether these motifs matched with the known motifs in the database including 24 species and 244 predicted RNA motifs [32] and found no motifs showing high homology with those reported here, indicating these four motifs were first identified in P. falciparum.

\section{Discussion}

In spite of the progress made in post-genomic research on malaria parasites, our knowledge on the function of small non-coding RNAs in these organisms remains insufficient. The limitation in cloning techniques to identify small RNAs in P. falciparum has restricted genome-wide small RNA characterization. Yet, high-throughput sequencing provides advanced depth and massive volumes, thereby enables the exploration of distinct categories of RNAs compared with traditional methods $[19,20]$. It is widely accepted that miRNAs are not the only regulators of gene expression; many other types of small RNAs have been identified to contribute to this process. tRFs is one such type, associated with many functions similar to miRNAs at the post-transcriptional level. Thus, it is possible that Plasmodium parasites complete gene expression regulation through molecules that can substitute for miRNAs, e.g. tRFs. Studies have shown that tRF expression is evolutionarily conserved from prokaryotes to mammalian cells. In the present study, we took advantage of high-throughput sequencing to obtain a coverage over 100 -fold for 18 $30 \mathrm{nt}$ length small RNAs, which provided sufficient depth for systematic analysis. Consistent with tRFs derived from plant or mammalian cell lines [13,33], we found three types of tRFs in P. falciparum: 5'ptRFs, 3'ptRFs and mid-ptRFs. Of these, 5 ptRFs is the most abundant class, while mid-ptRFs are generated from the $5^{\prime}$ end of the anticodon loop of their parental tRNAs, which has not been previously reported $[12,34,35]$.

The mechanism of tRF biogenesis has not been understood clearly because cleavage sites of known tRNA-specific nucleases remain ambiguous [36]. Information on the consensus feature(s) of cleavage sites on tRNAs needs to be fully addressed [37]. Therefore, we analyzed the motifs for the P. falciparum tRNAs to produce tRFs, and discovered four new motifs at the ends of the ptRFs that may aid the explanation of the versatile production of ptRFs. Intriguingly, motif 3 at the $3^{\prime}$ end of the mid-ptRFs and motif 4 at the $5^{\prime}$ end of the 3'ptRFs were found from different tRNA molecules in our analysis, while they shared analogous sequences. We noticed that these motifs are also situated at the same sites in those tRNAs not producing ptRFs. It is possible that the generation of ptRFs might involve multiple factors in addition to motif recognition. Moreover, the D-loop adjacent to motif 1 contains the "GG" conserved dinucleotide, which is required by RNase $\mathrm{P}$ and 39-tRNase processing and is highly conserved in Drosophila and humans [38]. Collectively, these findings provide a clue that there might be unidentified Plasmodium-derived endonucleases playing roles in the biogenesis of ptRFs.

The functional proteins closely associated with tRFs are reported to be DICER, AGO1, 2, 4 and 5 in plant cells, and AGO1, 3 and 4 in animal cells [13, 14, 33]. Yet, genome sequencing of $P$. falciparum has not revealed the existence of DICER and AGO homologs. Thus, the mechanism of function and related proteins 


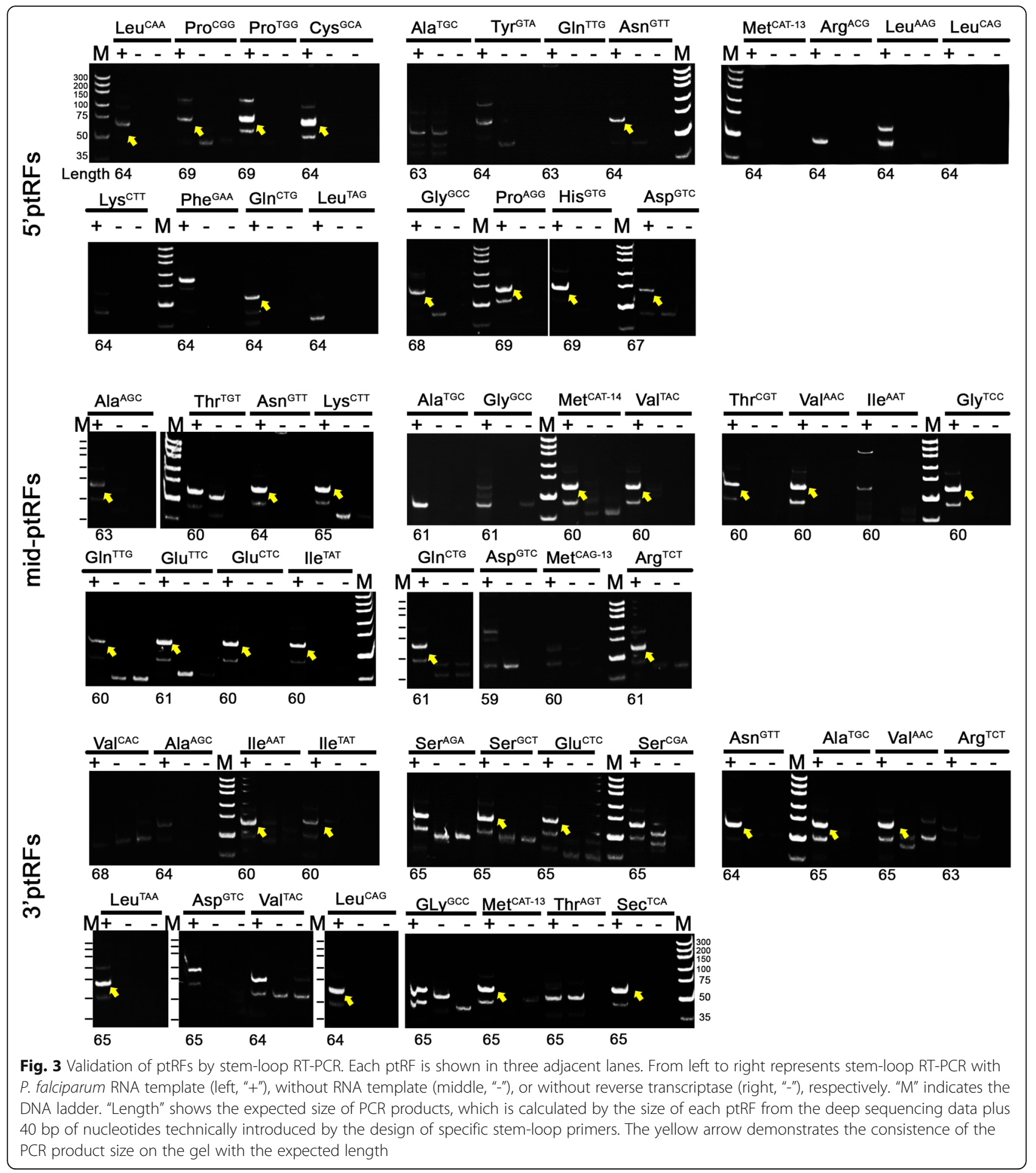

for ptRFs remains to be elucidated. Evidence from one previous study indicates that human miRNAs complexed with human AGO2 are translocated into the parasite cytoplasm to function as negative regulators of the infection [39]. Host-derived miRNA molecules have been shown to interfere with the synthesis of Plasmodium proteins [30]. Whether ptRFs function by binding to host AGO2 or other small RNA biogenesis-related apparatus should be further explored to strengthen our understanding of the interaction between malaria parasites and their human host.

In $P$. berghei and its closely related species $T$. gondii, half-tRNAs with a size of nearly 35 nt have been detected experimentally [40]. Although stress-induced 


\section{a}

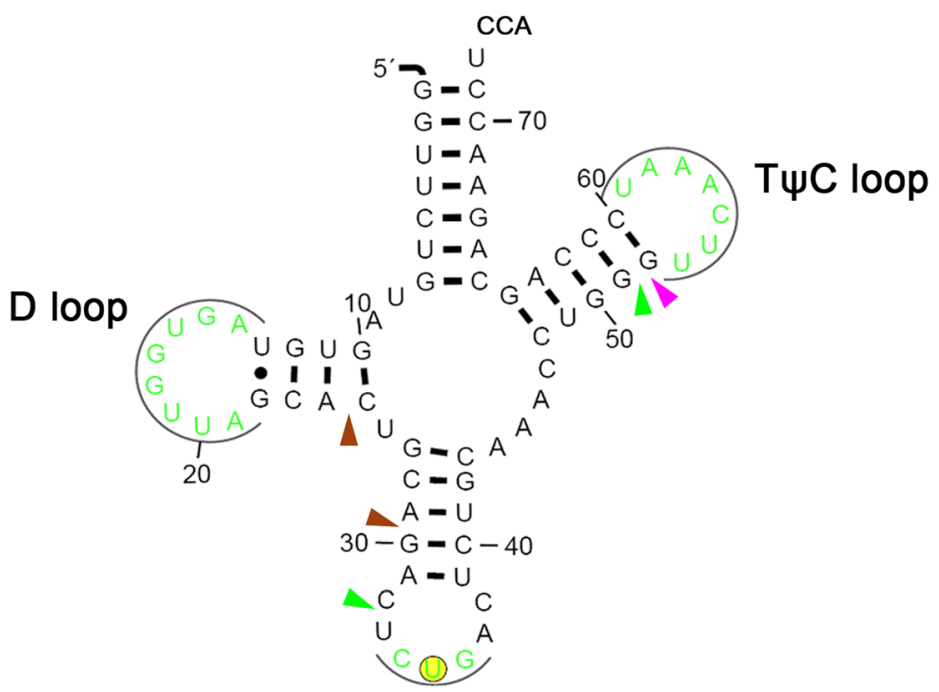

\section{Anti-codon loop}
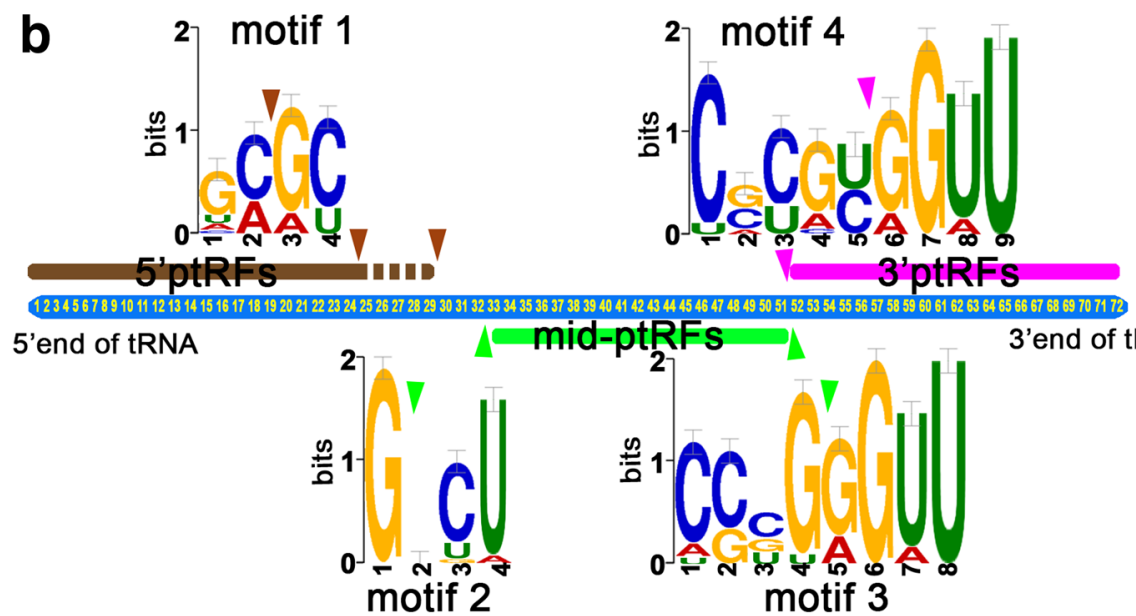

3'end of tRNA

Fig. 4 Cleavage sites and motif analysis of ptRFs. a Cleavage sites of ptRFs in mature tRNA, employing the secondary structure of tRNAs-GIn ${ }^{C T G}$ as an example. Brown arrowheads show the cleavage to generate 5 'ptRFs, green arrowheads demonstrate the cleavage sites for mid-ptRFs, and pink arrowhead indicates the cleavage points for 3'ptRFs. $\mathbf{b}$ Schematic representation of the motif sequences and locations at the cleavage sites of the ptRFs. Arrowheads are the same as for panel a

tRNA-halves are not included in this study, they need to be explored in P. falciparum. Therefore, more work is required to investigate ptRFs and other small RNAs as well as their function both in vitro and in vivo.

\section{Conclusions}

To our knowledge, this is the first comprehensive analysis of tRFs in P. falciparum. Three types of tRFs were identified, and four new motifs guiding their cleavage were revealed. The generation and function of these ptRFs need further exploration. Our work provides evidence of tRFs in P. falciparum, which could promote research on the functions of tRFs in the pathogenesis of
Plasmodium infections and potentially lead to new therapeutic approaches to malarial disease.

\section{Additional files}

Additional file 1: Table S1. Sequences of specific stem-loop reverse transcription primers. Table S2. Sequences of PCR primers for stem-loop RT-PCR. Table S3. RPM values and sizes of the top 20 abundant ptRFs for each type. (DOCX $41 \mathrm{~kb})$

Additional file 2: Figure S1. Abundance and correlation analysis of ptRFs. a Size distribution of ptRFs in total and unique categories. $\mathbf{b}$ Correlation of parental tRNAs of three types of ptRFs. Each dot represents a tRNA; R value indicates the Pearson's correlation coefficient. c Common parental tRNAs for the top 20 ptRFs of each type showing in Venn diagrams. (TIF 535 kb) 
Additional file 3: Figure S2. Size distributions of the 5'ptRFs in total and unique aspects. Solid boxes represent those with RPMs above $10^{5}$ and dotted boxes represent those with RPMs less than 5. (TIF $2602 \mathrm{~kb}$ )

Additional file 4: Figure S3. Size distributions of the mid-ptRFs in total and unique aspects. Dotted boxes correspond to those with RPMs less than 5. (TIF $2578 \mathrm{~kb}$ )

Additional file 5: Figure S4. Size distribution of the 3'ptRFs in total and unique aspects. The solid box symbolizes those with RPMs above $10^{5}$. (TIF 2626 kb)

\section{Abbreviations}

AGO: Argonaute protein; cDNA: Complementary DNA:

DNA: Deoxyribonucleic acid; endo-siRNA: Endogenous small interference RNA; gDNA: Genomic DNA; miRNA: MicroRNA; ncRNA: Non-coding RNA; Pf3D7: P. falciparum laboratory strain 3D7; piRNA: PiWi-interacting RNA; PiWi: P-element induced wimpy testis; ptRFs: Plasmodium tRFs; RBCs: Red blood cells; RNA: Ribonucleic acid; RPM: Relative number per one million reads; RPMI-1640: Roswell Park Memorial Institute-1640; rRNA: Ribosomal RNA; RT-PCR: Quantitative reverse-transcribed polymerase chain reaction; snoRNA: Small nucleolar RNA; snRNA: Small nuclear RNA; tRFs: Transfer RNAderived fragments; tRNA: Transfer RNA

\section{Acknowledgements}

We are grateful to Dr Huiliang Li from University College London for helpful discussions.

\section{Funding}

This work was supported by Beijing Natural Science Foundation (7173266), National Science and Technology Major Project (2018ZX10101-001) and CAMS Innovation Fund for Medical Sciences (CIFMS) (2017-I2M-3-016)

\section{Availability of data and materials}

The datasets generated and analyzed during the present study are available in the GEO repository under the accession number GSE119771.

\section{Authors' contributions}

ZSW, ZLW, HW, XH, CYW and WWD conceived and designed the experiments. ZSW and LHZ performed the experiments. ZSW and ZLW analyzed the data. ZSW, ZLW and HW wrote the manuscript. All authors read and approved the final manuscript.

\section{Ethics approval and consent to participate}

This work was approved by the Ethical Inspection Committee of the Institute of Basic Medical Sciences, Chinese Academy of Medical Sciences (no. ACUC-2017-056).

\section{Consent for publication}

Not applicable.

\section{Competing interests}

The authors declare that they have no competing interests.

\section{Publisher's Note}

Springer Nature remains neutral with regard to jurisdictional claims in published maps and institutional affiliations.

Received: 11 September 2018 Accepted: 7 January 2019

Published online: 15 January 2019

\section{References}

1. Li H, Li WX, Ding SW. Induction and suppression of RNA silencing by an animal virus. Science. 2002;296:1319-21.

2. Burenina OY, Elkina DA, Hartmann RK, Oretskaya TS, Kubareva EA. Small noncoding 6S RNAs of bacteria. Biochemistry (Mosc). 2015;80:1429-46.

3. Babski J, Maier LK, Heyer R, Jaschinski K, Prasse D, Jager D, et al. Small regulatory RNAs in Archaea. RNA Biol. 2014;11:484-93.

4. Okamura K. Diversity of animal small RNA pathways and their biological utility. WIREs RNA. 2012;3:351-68.

5. Borges F, Martienssen RA. The expanding world of small RNAs in plants. Nat Rev Mol Cell Biol. 2015;16:727-41.
6. Hamilton AJ, Baulcombe DC. A species of small antisense RNA in posttranscriptional gene silencing in plants. Science. 1999;286:950-2.

7. Portnoy V, Huang V, Place RF, Li LC. Small RNA and transcriptional upregulation. WIREs RNA. 2011;2:748-60.

8. Schopman NC, Heynen S, Haasnoot J, Berkhout B. A miRNA-tRNA mix-up: tRNA origin of proposed miRNA. RNA Biol. 2010;7:573-6.

9. Guo L, Liang T, Gu W, Xu Y, Bai Y, Lu Z. Cross-mapping events in miRNAs reveal potential miRNA-mimics and evolutionary implications. PLoS One. 2011;6:e20517.

10. Anderson $P$, Ivanov P. tRNA fragments in human health and disease. FEBS Lett. 2014:588:4297-304.

11. Goodarzi H, Liu X, Nguyen HC, Zhang S, Fish L, Tavazoie SF. Endogenous tRNA-derived fragments suppress breast cancer progression via YBX1 displacement. Cell. 2015;161:790-802.

12. Kumar P, Kuscu C, Dutta A. Biogenesis and function of transfer RNA-related fragments (tRFs). Trends Biochem Sci. 2016;41:679-89.

13. Kumar P, Anaya J, Mudunuri SB, Dutta A. Meta-analysis of tRNA derived RNA fragments reveals that they are evolutionarily conserved and associate with AGO proteins to recognize specific RNA targets. BMC Biology. 2014;12:78.

14. Loss-Morais G, Waterhouse PM, Margis R. Description of plant tRNA-derived RNA fragments (tRFs) associated with argonaute and identification of their putative targets. Biol Direct. 2013;8:6.

15. Keam SP, Young PE, McCorkindale AL, Dang TH, Clancy JL, Humphreys DT, et al. The human Piwi protein Hiwi2 associates with tRNA-derived piRNAs in somatic cells. Nucleic Acids Res. 2014;42:8984-95.

16. Cole C, Sobala A, Lu C, Thatcher SR, Bowman A, Brown JW, et al. Filtering of deep sequencing data reveals the existence of abundant Dicer-dependent small RNAs derived from tRNAs. RNA. 2009:15:2147-60.

17. WHO. World malaria report 2017. Geneva: World Health Organization; 2017.

18. Baum J, Papenfuss AT, Mair GR, Janse CJ, Vlachou D, Waters AP, et al. Molecular genetics and comparative genomics reveal RNAi is not functional in malaria parasites. Nucleic Acids Res. 2009:37:3788-98.

19. Xue $X$, Zhang $Q$, Huang $Y$, Feng $L$, Pan W. No miRNA were found in Plasmodium and the ones identified in erythrocytes could not be correlated with infection. Malar J. 2008;7:47.

20. Rathjen T, Nicol C, McConkey G, Dalmay T. Analysis of short RNAs in the malaria parasite and its red blood cell host. FEBS Lett. 2006;580:5185-8.

21. Couvillion MT, Sachidanandam R, Collins K. A growth-essential Tetrahymena Piwi protein carries tRNA fragment cargo. Genes Dev. 2010;24:2742-7.

22. Liao JY, Guo YH, Zheng LL, Li Y, Xu WL, Zhang YC, et al. Both endo-siRNAs and tRNA-derived small RNAs are involved in the differentiation of primitive eukaryote Giardia lamblia. Proc Natl Acad Sci USA. 2014;111:14159-64.

23. Reifur L, Garcia-Silva MR, Poubel SB, Alves LR, Arauco P, Buiar DK, et al, Distinct subcellular localization of tRNA-derived fragments in the infective metacyclic forms of Trypanosoma cruzi. Mem Inst Oswaldo Cruz. 2012;107: $816-9$.

24. Lambertz U, Oviedo Ovando ME, Vasconcelos EJ, Unrau PJ, Myler PJ, Reiner NE. Small RNAs derived from tRNAs and rRNAs are highly enriched in exosomes from both Old and New World Leishmania providing evidence for conserved exosomal RNA Packaging. BMC Genomics. 2015;16:151.

25. Trager W, Jensen JB. Human malaria parasites in continuous culture. Science. 1976;193:673-5.

26. Frith MC, Saunders NF, Kobe B, Bailey TL. Discovering sequence motifs with arbitrary insertions and deletions. PLoS Comput Biol. 2008;4:e1000071.

27. Gupta S, Stamatoyannopoulos JA, Bailey TL, Noble WS. Quantifying similarity between motifs. Genome Biol. 2007;8:R24.

28. Chen C, Ridzon DA, Broomer AJ, Zhou Z, Lee DH, Nguyen JT, et al. Realtime quantification of microRNAs by stem-loop RT-PCR. Nucleic Acids Res. 2005;33:e179.

29. Kramer MF. Stem-loop RT-qPCR for miRNAs. Curr Protoc Mol Biol. 2011; Chapter 15:Unit 15.10

30. LaMonte G, Philip N, Reardon J, Lacsina JR, Majoros W, Chapman L, et al. Translocation of sickle cell erythrocyte microRNAs into Plasmodium falciparum inhibits parasite translation and contributes to malaria resistance. Cell Host Microbe. 2012;12:187-99.

31. Wang J, Liu X, Jia B, Lu H, Peng S, Piao X, et al. A comparative study of small RNAs in Toxoplasma gondii of distinct genotypes. Parasit Vectors. 2012; $5: 186$.

32. Ray D, Kazan H, Cook KB, Weirauch MT, Najafabadi HS, Li X, et al. A compendium of RNA-binding motifs for decoding gene regulation. Nature. 2013;499:172-7. 
33. Alves CS, Vicentini R, Duarte GT, Pinoti VF, Vincentz M, Nogueira FT. Genome-wide identification and characterization of tRNA-derived RNA fragments in land plants. Plant Mol Biol. 2017;93:35-48.

34. Gebetsberger J, Zywicki M, Kunzi A, Polacek N. tRNA-derived fragments target the ribosome and function as regulatory non-coding RNA in Haloferax volcanii. Archaea. 2012;2012:260909.

35. Diebel KW, Zhou K, Clarke AB, Bemis LT. Beyond the Ribosome: Extratranslational functions of tRNA fragments. Biomark Insights. 2016;1 (Suppl. 1):1-8.

36. Saida F, Odaert B. RNA recognition and cleavage by sequence-specific endoribonucleases. Protein Pept Lett. 2007;14:103-11.

37. Lee YS, Shibata Y, Malhotra A, Dutta A. A novel class of small RNAs: tRNAderived RNA fragments (tRFs). Genes Dev. 2009;23:2639-49.

38. Levinger L, Bourne R, Kolla S, Cylin E, Russell K, Wang X, et al. Matrices of paired substitutions show the effects of tRNA D/T loop sequence on Drosophila RNase $P$ and 3'-tRNase processing. J Biol Chem. 1998:273:1015-25.

39. Wang Z, Xi J, Hao X, Deng W, Liu J, Wei C, et al. Red blood cells release microparticles containing human argonaute 2 and miRNAs to target genes of Plasmodium falciparum. Emerg Microbes Infect. 2017;6:e75.

40. Galizi R, Spano F, Giubilei MA, Capuccini B, Magini A, Urbanelli L, et al. Evidence of tRNA cleavage in apicomplexan parasites: Half-tRNAs as new potential regulatory molecules of Toxoplasma gondii and Plasmodium berghei. Mol Biochem Parasitol. 2013;188:99-108,

Ready to submit your research? Choose BMC and benefit from:

- fast, convenient online submission

- thorough peer review by experienced researchers in your field

- rapid publication on acceptance

- support for research data, including large and complex data types

- gold Open Access which fosters wider collaboration and increased citations

- maximum visibility for your research: over $100 \mathrm{M}$ website views per year

At $\mathrm{BMC}$, research is always in progress.

Learn more biomedcentral.com/submissions 